\title{
Combining lipidomics and machine learning to measure clinical lipids in dried blood spots
}

\author{
Stuart G. Snowden ${ }^{1}\left[\right.$ - Aniko Korosi ${ }^{2} \cdot$ Susanne R. de Rooij ${ }^{3,4} \cdot$ Albert Koulman $^{1}$
}

Received: 9 March 2020 / Accepted: 11 July 2020 / Published online: 24 July 2020

(c) The Author(s) 2020

\begin{abstract}
Introduction Blood-based sample collection is a challenge, and dried blood spots (DBS) represent an attractive alternative. However, for DBSs to be an alternative to venous blood it is important that these samples are able to deliver comparable associations with clinical outcomes. To explore this we looked to see if lipid profile data could be used to predict the concentration of triglyceride, HDL, LDL and total cholesterol in DBSs using markers identified in plasma.

Objectives To determine if DBSs can be used as an alternative to venous blood in both research and clinical settings, and to determine if machine learning could predict 'clinical lipid' concentration from lipid profile data.

Methods Lipid profiles were generated from plasma $(n=777)$ and DBS $(n=835)$ samples. Random forest was applied to identify and validate panels of lipid markers in plasma, which were translated into the DBS cohort to provide robust measures of the four 'clinical lipids'.

Results In plasma samples panels of lipid markers were identified that could predict the concentration of the 'clinical lipids' with correlations between estimated and measured triglyceride, HDL, LDL and total cholesterol of $0.920,0.743,0.580$ and 0.424 respectively. When translated into DBS samples, correlations of $0.836,0.591,0.561$ and 0.569 were achieved for triglyceride, HDL, LDL and total cholesterol.
\end{abstract}

Conclusion DBSs represent an alternative to venous blood, however further work is required to improve the combined lipidomics and machine learning approach to develop it for use in health monitoring.

Keywords Lipidomics · Total cholesterol $\cdot$ Triglyceride $\cdot$ HDL $\cdot$ LDL

Electronic supplementary material The online version of this article (https://doi.org/10.1007/s11306-020-01703-0) contains supplementary material, which is available to authorized users.

Albert Koulman

ak675@medschl.cam.ac.uk

1 Core Metabolomics and Lipidomics Laboratory, Metabolic Research Laboratories, Institute of Metabolic Science, University of Cambridge, Level 4 Pathology, Cambridge Biomedical Campus, Cambridge CB2 0QQ, UK

2 Centre for Neuroscience, Swammerdam Institute for Life Sciences, University of Amsterdam, Amsterdam, The Netherlands

3 Department of Clinical Epidemiology, Amsterdam University Medical Centre, Biostatistics \& Bio informaticslocation AMC, Amsterdam, The Netherlands

4 Department of Public Health, Amsterdam University Medical Center, University of Amsterdam, Amsterdam, The Netherlands

\section{Introduction}

Traditionally, in high-income countries there is significant infrastructure to facilitate preclinical population health monitoring and research that is not present in low income countries leaving a significant unmet need for research and health monitoring (Kreuter et al. 2016; Johannessen 2010). Collecting samples in a hospital setting is still relatively straight forward, however owing to the difficulty, expense (relative to income) and risk of infection only individuals with serious or life-threatening symptoms are usually taken to hospital meaning that a sample cohort collected in a hospital alone would be biased.

In low income settings sending teams into the community to collect samples is one part of the solution to this. However, venous blood samples will need to be processed and frozen within a few hours of sample collection (Yin et al. 2015) and in rural areas of an LIC access to laboratory facilities to do this is unlikely to be available. It is with 
these limitations in mind that dried blood spots represent an attractive alternative to venous blood collection as it enables samples to be collected in remote geographic locations because processing the sample for storage does not require laboratory facilities (Spooner et al. 2019; Li and Tse 2010). The ability to collect smaller blood volumes is also attractive for working with fragile patient populations in LIC settings e.g. malnourished infants (Spooner et al. 2019; Lakshmy et al. 2014; Komada et al. 2015). In addition to this Prentice et al. (Prentice et al. 2013) showed that whilst room temperature storage of DBS samples for two years does lead to significant differences in metabolite composition, room temperature storage for just a week has very little effect, meaning that there can be a short delay between sample collection and storage at either $-20^{\circ} \mathrm{C}$ or $-80{ }^{\circ} \mathrm{C}$. These findings are consistent with other studies that have shown that a range of analytes including lipids, HIV antibodies and drugs including ceftriaxone and cocaine are stable on DBS cards stored at room temperature for short periods (Kyle et al. 2017; Page-Sharp et al. 2016; Yassin et al. 2013; Alfazil and Anderson 2008). In the context of research, where large sample cohorts can take several years to collect and especially when repeat sampling is involved, the effect of long term storage at $-80{ }^{\circ} \mathrm{C}$ is also an important experimental factor to consider. Prentice et al. also looked at the stability of lipid analytes in DBS stored at both $-20{ }^{\circ} \mathrm{C}$ and $-80{ }^{\circ} \mathrm{C}$ and demonstrate that storage at $-80{ }^{\circ} \mathrm{C}$ for 2 years had a minimal effect on the lipid composition of the DBS samples (Prentice et al. 2013).

For both research and health care monitoring it is important to determine if there is good concordance in the concentrations measured in both DBS and plasma samples. There is a significant body of literature looking at how to analyse a wide range of drug analytes in these samples (Wilhelm et al. 2014; Elst et al. 2013; Manicke et al. 2011), with DBS samples being standardly used pharmokinetic studies in neonates and young children (Abu-Rabie and Spooner 2009). An array of endogenous compounds have also been measured from dried blood spots including $\mathrm{HbA} 1 \mathrm{c}$ (Crimmins et al. 2014; Lacher et al. 2013), total cholesterol (Abhijit et al. 2016; Miller et al. 2015; Corso et al. 2016), HDL (Lacher et al. 2013; Miller et al. 2015; Samuelsson et al. 2015), triglyceride (Lakshmy et al. 2012,2010; Quraishi et al. 2006) and CRP (Elst et al. 2013) among others. A detailed discussion of this literature is beyond the scope of this manuscript and there are several excellent reviews on this subject (Spooner et al. 2019; Zakaria et al. 2016; Chance et al. 2014).

Many of these studies have been successful and achieved good concordance between the concentration of a given analyte when measured in both DBS and plasma samples making them useful tools in research and healthcare monitoring. For example Lakshmy et al. was able to achieve a correlation of $r=0.797$ between concentrations of total cholesterol measured in DBS and serum samples (Lakshmy et al. 2012). This is broadly in keeping with the other results reported in the literature with Samuelsson et al. reporting $r=0.480$ (Samuelsson et al. 2015), Corso et al. achieving $r=0.611$ (Corso et al. 2016) and Lacher et al. an $R^{2}$ of 0.340 (Lacher et al. 2013) between concentrations of total cholesterol measured in DBS and serum samples. This concordance between studies is not just seen for total cholesterol with Quraishi et al. obtaining a correlation of $r=0.970$ between triglyceride concentration measured in DBS and plasma samples (Quraishi et al. 2006) with subsequent studies achieving correlations of $r=0.838$ and $r=944$ (Lakshmy et al. 2012,2010). The ability to obtain a good measure of a given analyte with a strong correlation between plasma and DBS concentrations is not the only factor in ensuring that the two sample types are comparable; it is also important to ensure that the relationship between a given analyte and a clinical outcome measure present in the original blood sample are maintained in the DBS sample. For example if we consider measurement of activity of steraoyl-CoA desaturase using the ratio of $\mathrm{PC}(32: 1)$ to $\mathrm{PC}(32: 0)$, and either of $\mathrm{PC}(32: 1)$ or $\mathrm{PC}(32: 0)$ are diffuse differently across the sorbent or have differing extraction recoveries then this ratio will be changed giving false reading for the activity of this enzyme.

With this in mind the aims of this study were twofold. Firstly, we aimed to determine the comparability of results obtained from interrogating complex lipidomics data generated from DBS and plasma samples. We will do this by using random forest machine learning, which will be applied to lipid profile data generated from human plasma samples to predict the circulating concentration of four clinically important lipoproteins, triglyceride, HDL, LDL and total cholesterol. Subsequently we will measure these biomarkers in DBS samples and determine if these biomarkers can predict the concentration of these lipoproteins. We have chosen to use samples from the Dutch famine birth cohort and the Amsterdam Born Children and their Development cohorts rather than freshly collected samples as this better reflects the research cohorts that these developed approaches will be applied to. We have chosen to work with dried blood spot samples where the concentration of these lipoproteins was measured in a paired capillary blood sample rather than measured directly from the DBS. We have made this decision as we specifically want to DBS lipid profile data to predict the concentrations of these lipoproteins in blood and whilst measuring them in the DBS sample would provide an accurate surrogate of the blood concentration measuring it directly from the blood is preferable. Our second aim was to see if the estimated concentrations that we obtained from the DBS samples were accurate enough to be used in clinical practice and thus the bias of health care monitoring. 


\section{Materials and methods}

\subsection{Sample description}

\subsubsection{Dutch Famine Birth Cohort (DFBC)}

The Dutch Famine Birth Cohort is a historical cohort of 2,414 men and women born in the Wilhemina Gasthuis hospital in Amsterdam between the 1st of November 1943 and 28th of February 1947. The selection procedures of this cohort have been described in detail previously (Lumey et al. 1993). In the present study, we analysed 777 plasma samples collected between 2002 and 2004 from individuals aged 55.7 to 60.8 years old, with all samples stored at $-80^{\circ} \mathrm{C}$ prior to analysis. The study was approved by the local Medical Ethics committee and conducted in accordance with the Helsinki declaration. All participants gave written informed consent.

\subsubsection{Amsterdam Born Children and their Development $(A B C D)$ cohort}

The Amsterdam Born Children and their Development cohort is a large prospective birth cohort established in 2003-2004 in which mothers, fathers and their children are followed from pregnancy to adulthood, examining parental factors and their influence on the child's health and development (Eijsden et al. 2011). Dried blood spots were collected in 2015-2016 when the children were between 11 and 13 years old with samples collected by numbing the participants finger before pricking it and dropping blood onto Whatman 903 cards filled with a solution of $5 \mathrm{mg}$ butylated hydroxytoluene (BHT) per $10 \mathrm{ml}$ ehthanol. Samples were subsequently dried at room temperature, catalogued and stored at $-80{ }^{\circ} \mathrm{C}$ prior to analysis. Lipid profiles were generated from 835 dried blood spot samples collected from children aged between 11.1 and 13.0 years (Eijsden et al. 2011). The study was approved by the local Medical Ethics committee and conducted in accordance with the Helsinki declaration. All participants and their parents gave written informed consent.

\subsection{Measurement of 'clinical lipids'}

In the Dutch Famine Birth Cohort, fasting blood was drawn for analysis of Low Density Lipoprotein (LDL)-cholesterol, High Density Lipoprotein (HDL)-cholesterol and triglyceride. HDL-cholesterol and triglyceride were measured using an enzymatic colorimetric reagent test using a P-800 Modular (Roche/Hitachi), with LDL-cholesterol calculated using the Friedewald formula and total cholesterol determined by combining the concentration of HDL and LDL cholesterol (Lumey et al. 1993). In the ABCD cohort samples were collected after a $3 \mathrm{~h}$ fasting period with capillary blood from a finger prick was collected within a capillary tube within $10 \mathrm{~s}$ with the sample subsequently transferred to the test cassette with TG, TC, HDL, and LDL concentration determined using the Cholestec LDX Analyzer (Eijsden et al. 2011).

\subsection{Preparation of plasma samples}

Samples were extracted from plasma (which was stored at $-80{ }^{\circ} \mathrm{C}$ until assay) as described previously (Harshfield et al. 2019). In short: $100 \mu$ l of LC-MS grade water and $150 \mu \mathrm{l}$ of internal standard mix (Table S1) were added to $15 \mu$ of plasma in a 96 well glass coated plate prior to mixing for $10 \mathrm{~s}$. Subsequently, $750 \mu \mathrm{l}$ of LC-MS grade methyltertiary butyl ether (MTBE) and a further $200 \mu$ of LC-MS grade water were added to each well before shaking for $10 \mathrm{~s}$. Once mixed, plates were spun at $845 \times g$ for 2 min to achieve phase separation with $25 \mu \mathrm{l}$ of the upper organic phase transferred to a new glass coated plate with $90 \mu \mathrm{l}$ of MS-mix (7.5 mM ammonium acetate in IPA: $\mathrm{CH}_{3} \mathrm{OH} 2: 1$ ), which was subsequently added to each well.

\subsection{Preparation of dried blood spot samples}

Dried blood spot samples were prepared as described previously (Koulman et al. 2014). In short: $100 \mu \mathrm{l}$ of LC-MS grade water and $150 \mu \mathrm{l}$ of internal standard mix (Table S1) were added to a $3.2 \mathrm{~mm}$ diameter dried blood spot in a 96 well glass coated plate prior to mixing for $10 \mathrm{~s}$. Subsequently, $750 \mu \mathrm{l}$ of LC-MS grade methyl-tertiary butyl ether (MTBE) and a further $200 \mu \mathrm{l}$ of LC-MS grade water were added to each well before shaking for $10 \mathrm{~s}$. Once mixed, plates were spun at $845 \times g$ for 2 min to achieve phase separation with $150 \mu \mathrm{l}$ of the upper organic phase transferred to a new glass coated plate and was dried down under a continuous stream of nitrogen. The dried samples were then reconstituted in $25 \mu \mathrm{l}$ of MTBE to which $90 \mu \mathrm{l}$ of MS-mix (7.5 mM ammonium acetate in IPA:CH3OH 2:1) was subsequently added to each well.

\subsection{Preparation of quality control samples}

Three levels of quality controls samples $100 \%$ serum, 50:50 $(v: v)$ serum:water and 25:75 (v:v) serum:water were prepared from a pooled serum sample with $15 \mu$ l extracted following the same procedure applied to DBS and plasma samples. In addition to being used to assess and correct plate to plate variance the quality control levels are also used to aid signal identification with real signals showing a linear increase in abundance with an increase in serum concentration. 


\subsection{DIMS lipidomic profiling}

Samples were infused into an Exactive Orbitrap (Thermo, Hemel Hempstead, UK) using a Triversa Nanomate (Advion, Ithaca, USA). Data collection began $20 \mathrm{~s}$ after the infusion began, initially analysing samples in the positive ionisation mode with an ionisation voltage of $1.2 \mathrm{kV}$ applied. Data was acquired between 150 and $2000 \mathrm{~m} / z$ with a scan rate of $1 \mathrm{~Hz}$ giving a mass resolution of 65,000 at $400 \mathrm{~m} / z$. A more detailed description of the instrument parameters can be found in Harshfield et al. (2019).

\subsection{Processing of lipidomic data}

Raw data files were converted to.mzXML files using msConvert (ProteoWizard) (Chambers et al. 2012), and were subsequently processed in $\mathrm{R}$ (version 3.2.2) using an in house script to compare spectra against a list of 1649 lipid species, with a relative intensity and mass deviation value recorded for each lipid in every sample. We applied 4 filtering steps for quality control of the data and focus subsequent analysis on analytically robust signals. The first step was to remove lipids with a mean mass deviation between expected and recorded mass of greater than $5 \mathrm{ppm}$, the second step was to remove signals with an average intensity in the samples less than 5 times greater than in the blanks. The third step was to remove signals with 0 values in greater than $10 \%$ of samples. The final step was to remove lipids with an $r<0.9$ in our QC dilution series.

In this study, the 'discovery' and 'validation' cohort were processed in parallel to ensure that datasets contained the same lipid variables. To control for potential differences in the volume of blood sampled from each blood spot all data was normalised to total signal intensity, this was done by calculating the total abundance of all of the lipid signals within a given sample then dividing the abundance of each of these signals by the total. After normalisation data was subsequently mean centred to allow for a direct comparison between the two cohorts. The data in the DBS cohort was filtered using the same steps as the plasma samples but was processed independently of the plasma samples as the composition of DBS samples will be different to that of plasma samples (Table 1).

\subsection{Statistical analysis}

Data analysis was performed for each of the four 'clinical lipid' markers (triglyceride, LDL, HDL and total cholesterol) independently. The analysis was performed in two stages, in the first stage of the analysis, we applied a random forest model to all of the data in the 'discovery' cohort (the DFBC) to identify a panel of lipid biomarkers capable of robustly predicting the concentration of the clinical biomarker. This
Table 1 Clinical characteristics of the sample cohorts used in this study

\begin{tabular}{lll}
\hline & $\begin{array}{l}\text { Dutch famine } \\
\text { birth cohort }\end{array}$ & $\begin{array}{l}\text { Amsterdam born children } \\
\text { and their development } \\
\text { cohort }\end{array}$ \\
\hline Sample N $^{\mathrm{o}}$ & 777 & 835 \\
Gender (\% female) & $54.2 \%$ & $50.6 \%$ \\
Age (years) & $58.3 \pm 1.0$ & $11.8 \pm 0.4^{* * *}$ \\
BMI & $28.6 \pm 4.9$ & $17.6 \pm 2.5 * * *$ \\
TriG (mmol/L) & $1.53 \pm 1.00$ & $0.97 \pm 0.54^{* * *}$ \\
HDL (mmol/L) & $1.51 \pm 0.42$ & $1.48 \pm 0.32^{* * *}$ \\
LDL (mmol/L) & $3.63 \pm 1.00$ & $2.17 \pm 0.55^{* * *}$ \\
Total Cholesterol & $5.83 \pm 1.08$ & $4.09 \pm 0.65^{* * *}$ \\
$(\mathrm{mmol} / \mathrm{L})$ & & \\
\hline
\end{tabular}

$B M I$ body mass index, $H D L$ high density lipoprotein, $L D L$ low density lipoprotein, $\mathrm{Tri} G$ triglyceride

$* * *<0.0001$

was done by splitting the samples into training and test sets (samples split 70:30 respectively) and calculating a random forest model in the training set and assessing its performance in the test set. To determine the number of lipids to include in the predictive panel, iterative random forest models were calculated using the highest ranked variable from the 'all data' model first and then adding additional variables to each model (one at the time) until we achieved a model that performed as well as the model calculated using all lipids.

In the second stage, we determined if the panel of lipid biomarkers identified in the plasma samples from the DFBC could be used to predict the concentration of the clinical markers in the 'validation' cohort. This was done by dividing the DBS cohort (ABCD cohort) into a training and test set (70:30) and calculating a random forest model in the training set and assessing its predictive performance in the testing set based on the correlation between the measured and predicted concentration of the clinical biomarker.

The relationships between individual lipid species and given clinical biomarker concentrations were determined using generalised linear models (GLM) applied to the whole of the dataset. All models were calculated in ' $\mathrm{R}$ ' (version 3.4.2). Controlling for type 1 errors was performed by determining if $p$-values passed a Bonferroni adjusted significance threshold of $p=0.0004$ calculated based on all 125 lipids measured in this study.

\section{Results}

In plasma samples 163 lipids from 11 classes passed quality control procedures, whilst 118 lipids from 11 classes were identified in the dried blood spot samples with $71 \%$ of the lipids measured in DBS also measured in plasma. 


\subsection{Estimation of triglycerides concentration}

A panel of 12 lipids (Table S2) produced a model with a mean square of residual (MSR) of 0.199 in the training set (Table S2) and a correlation of $r=0.914$ and $p=7.0 \times 10^{-92}$ between estimated and measured triglyceride in the test set (Fig. 1a). With the exception of DG(34:2) and DG(36:2), which were not detected in lipid profiles generated from DBS samples the remaining 10 lipids were used to generate a random forest model with an MSR of 0.111 in the training set (Table S2), with estimated and measured triglyceride correlating with $\mathrm{r}=0.836$ and $\mathrm{p}=1.9 \times 10^{-63}$ in the test set (Fig. 1b). The samples in the test set of the DBS cohort were divided into quartiles based on their measured and their predicted TriG independently with the number of samples that were in the same quartile for both measured and predicted TriG concentration being recorded. The percentage overlap was higher in all quartiles than the $25 \%$ that we would expect to see by chance. It is also interesting to note that there was a higher percentage overlap in the 1st and 4th quartiles compared to the overlap in the 2nd and 3rd quartiles (Table 2).

\subsection{Estimation of HDL concentration}

In the discovery cohort a panel of 11 lipids (Table S3) was identified that generated a random forest model with an MSR of 0.082 in the training set (Table S2), with a correlation of $r=0.748$ and $p=4.5 \times 10^{-42}$ between estimated and measure HDL in the test set (Fig. 2a). All 11 of the lipids of panel identified in the discovery cohort of plasma samples were measured in the DBS samples with these lipid used to generate a random forest model with an MSR of 0.068 in the training set (Table S2), with a correlation of $r=0.591$ and $p=9.4 \times 10^{-21}$ between estimated and measure HDL concentration in the test set (Fig. 2b).

\subsection{Estimation of LDL concentration}

In plasma samples random forest identified a 10 lipid panel of markers (Table S4) with an MSR of 0.811 in the training set (Table S2), with an estimated and measured LDL concentration correlating with $\mathrm{r}=0.556$ and $\mathrm{p}=5.1 \times 10^{-21}$ in the test set (Fig. 3a). In the DBS samples from the ABCD cohort $\mathrm{CE}(14: 0)$ and $\mathrm{TG}(47: 4)$ were not detected in the lipid profiles generated from DBS samples.A random forest model generated using the 8 remaining lipids from the predictive panel with an MSR of 0.231 in the training set (Table S2), and a correlation of $r=0.561$ and $p=4.6 \times 10^{-21}$ between estimated and measured LDL concentration in the test set (Fig. 3b).

The quartile distribution of LDL showed that the percentage overlap was higher in all quartiles than the $25 \%$ that we would expect to see by chance (Table 2).

\subsection{Estimation of total cholesterol concentration}

A panel of 11 lipids (Table S5) were used to generate a random forest model with an MSR of 1.05 in the training set (Table S2), and a correlation of $\mathrm{r}=0.424$ and $\mathrm{p}=1.8 \times 10^{-11}$ between estimated and measured total cholesterol in the test set (Fig. 4a). In the ABCD cohort, LPC(20:4), CE(14:0), $\mathrm{CE}(16: 1)$ and $\mathrm{PC}-\mathrm{O}(40: 5)$ which form part of the predictive panel identified in plasma samples were not detected in the lipid profiles generated from DBS samples. A random forest model generated using the remaining 7 lipids with an MSR of 0.288 in the training set (Table S2), with estimated and measured total cholesterol concentration correlating with $r=0.569$ and $p=9.3 \times 10^{-22}$ (Fig. 4b). Again, as with the quartile distribution of the other lipids, the percentage overlap for total cholesterol was higher in all quartiles than the $25 \%$ that we would expect to see by chance (Table 2).
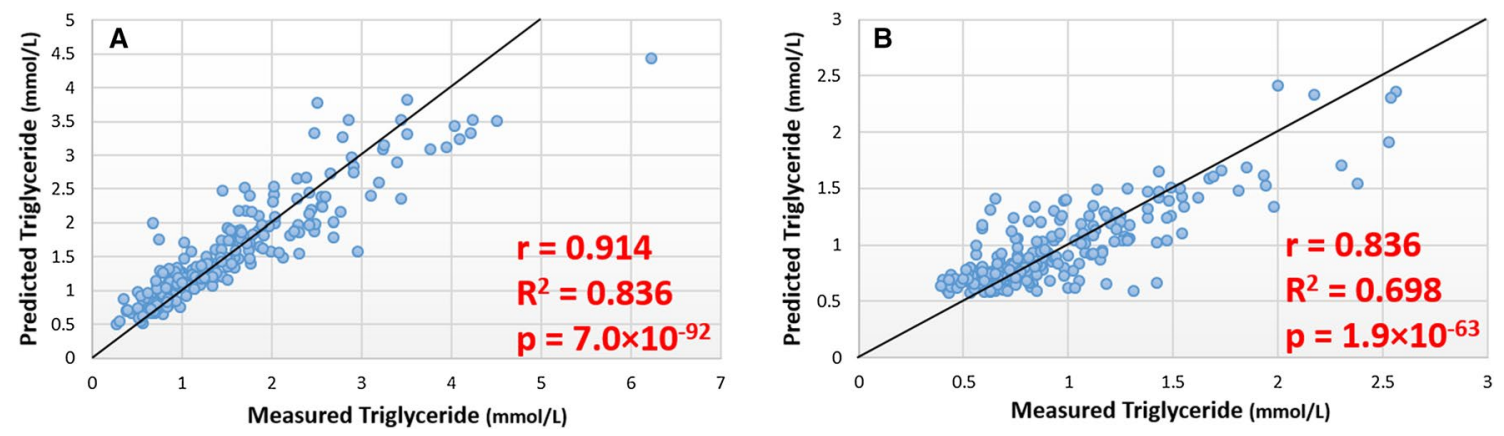

Fig. 1 Scatter plots showing predicted vs. measured Triglyceride concentration in discovery and validation analysis. a Results of model trained and tested in 'discovery' cohort. b Results of models trained and tested in DBS 'validation' cohort using lipid markers identified in plasma 
Table 2 Comparison of relative distribution based on quartiles of measured clinical lipid concentration and the concentration predicted from dried blood spot samples

\begin{tabular}{|c|c|c|c|c|c|}
\hline \multicolumn{6}{|c|}{ Dutch Famine Birth Cohort (plasma) } \\
\hline & & 'Desirable' & 'Borderline' & 'Poor’ & \\
\hline \multirow[t]{2}{*}{ Triglyceride } & Overlap* & 144 & 16 & 34 & \\
\hline & $\%$ Overlap & 90.5 & 64.0 & 72.3 & \\
\hline \multirow[t]{2}{*}{ HDL } & Overlap* & 82 & 23 & 45 & \\
\hline & $\%$ Overlap & 73.2 & 56.1 & 57.7 & \\
\hline \multirow[t]{2}{*}{ LDL } & Overlap* & 30 & 147 & 13 & \\
\hline & $\%$ Overlap & 85.7 & 89.1 & 41.9 & \\
\hline \multirow[t]{2}{*}{ Total Cholesterol } & Overlap* & 3 & 135 & 12 & \\
\hline & $\%$ Overlap & 100.0 & 64.6 & 63.1 & \\
\hline \multicolumn{6}{|c|}{ Amsterdam Born Children and their Development Cohort (dried blood spots) } \\
\hline & & 1st Quartile & 2nd Quartile & 3rd Quartile & 4th Quartile \\
\hline \multirow[t]{2}{*}{ Triglyceride } & Overlap* & 36 & 32 & 32 & 44 \\
\hline & $\%$ Overlap & 60.0 & 53.3 & 53.3 & 73.3 \\
\hline \multirow[t]{2}{*}{ HDL } & Overlap* & 37 & 24 & 21 & 35 \\
\hline & $\%$ Overlap & 61.7 & 40.0 & 35.0 & 58.3 \\
\hline \multirow[t]{2}{*}{ LDL } & Overlap* & 34 & 20 & 22 & 36 \\
\hline & $\%$ Overlap & 56.7 & 33.3 & 36.7 & 60.0 \\
\hline \multirow[t]{2}{*}{ Total Cholesterol } & Overlap* & 35 & 21 & 20 & 34 \\
\hline & \% Overlap & 58.3 & 35.0 & 33.3 & 56.7 \\
\hline
\end{tabular}

Overlap* the number of samples with the measured and predicted lipid concentrations falling in the same quartile. Triglyceride 'Desirable' $<1.7 \mathrm{mmol} / \mathrm{l}$, 'Borderline' 1.7-2.2 mmol/l, 'Poor' $>2.2 \mathrm{mmol} / \mathrm{l}$, HDL 'Desirable' $>1.5 \mathrm{mmol} / \mathrm{l}$, 'Borderline' 1.5-1.1 mmol/l, 'Poor' $<1.2 .2 \mathrm{mmol} / \mathrm{l}$, LDL 'Desirable' $<2.6 \mathrm{mmol} / \mathrm{l}$, 'Borderline' 2.6-5.0 mmol/l, 'Poor' $>5.0 \mathrm{mmol} / \mathrm{l}$, Total cholesterol 'Desirable' $<5.2 \mathrm{mmol} / \mathrm{l}$, 'Borderline' $5.2-6.2 \mathrm{mmol} / \mathrm{l}$, 'Poor' $>6.2 \mathrm{mmol} / \mathrm{l}$
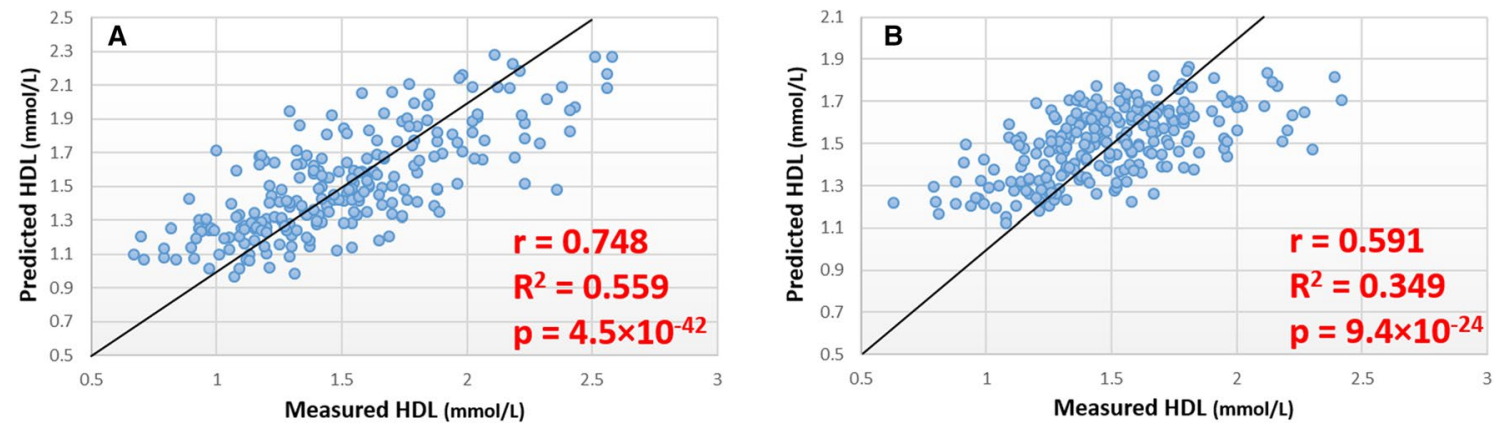

Fig. 2 Scatter plots showing predicted vs. measured HDL concentration in discovery and validation analysis. a Results of model trained and tested in 'discovery' cohort. b Results of models trained and tested in DBS 'validation' cohort using lipid markers identified in plasma

\subsection{Univariate analysis of lipid associations with lipoprotein concentration}

Of the 44 lipid associations from the 4 predictive panels $8(18 \%)$ were not measured in the DBS samples whilst 36 $(82 \%)$ were successfully validated with a strong correlation $(r=0.917)$ observed between the correlation coefficients measured calculated between the individual lipid abundance and lipoprotein concentration in both plasma and DBS samples.

Of the 12 lipids used to predict triglyceride concentration all significantly associated with TriG concentration passing bonferroni correction at $(\mathrm{p}<0.0004)$ in both DFBC and ABCD cohorts, except for DG(34:2) and DG(36:2) which were not measured in DBS samples (Table S2). 

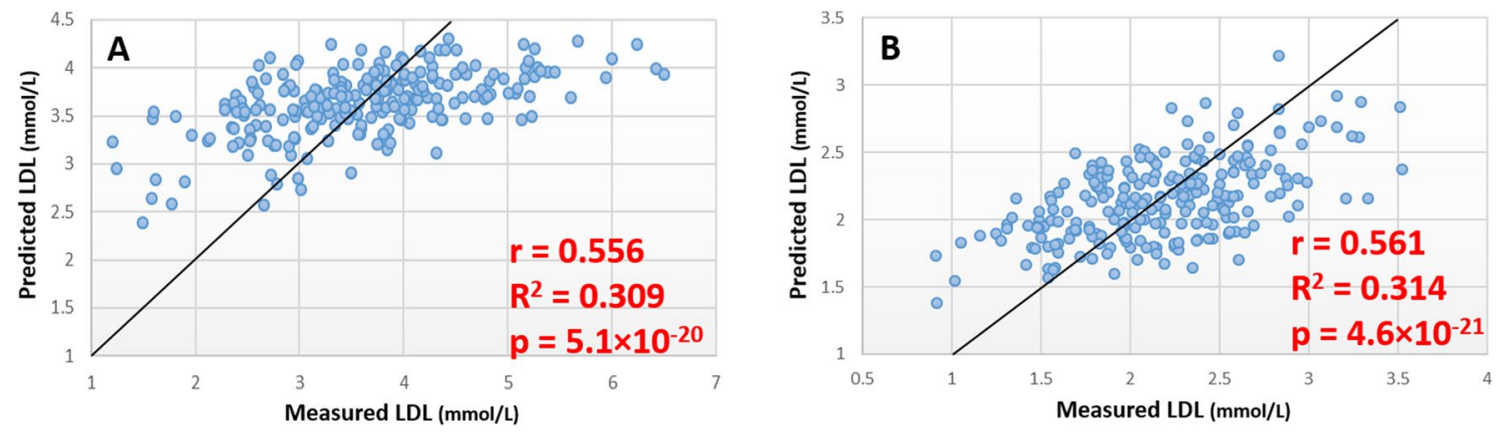

Fig. 3 Scatter plots showing predicted vs. measured LDL concentration in discovery and validation analysis. a Results of model trained and tested in 'discovery' cohort. b Results of models trained and tested in DBS 'validation' cohort using lipid markers identified in plasma

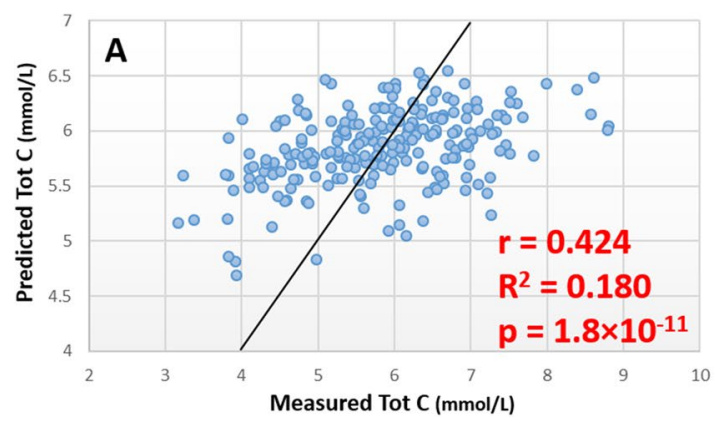

Fig. 4 Scatter plots showing predicted vs. measured total cholesterol concentration in discovery and validation analysis. a Results of model trained and tested in 'discovery' cohort. b Results of models trained

With the exception of PC(38:5) all significantly associated with HDL concentration passing bonferroni correction at $(\mathrm{p}<0.0004)$ in both DFBC and ABCD cohorts (Table S3). Whilst not passing the bonferroni corrected p-threshold PC $(38: 5)$ was significantly $(\mathrm{p}<0.05)$ associated with HDL concentration in both plasma and DBS samples (Table S3).

With the exception of CE(14:0) and TG(47:4) which were not measured in dried blood spot samples all lipids were significantly associated with LDL concentration in both sample types passing a bonferroni correct p-threshold $(\mathrm{p}<0.0004)$ (Table S4).

Of the 11 lipids total cholesterol concentration all were significantly $(\mathrm{p}<0.05)$ associated with total cholesterol concentration however only 7 passed a bonferroni correct $\mathrm{p}$-threshold $(\mathrm{p}<0.0004)$ with $\mathrm{CE}(18: 2)$, PC-O(34:1), PC-O(40:5) and TG(56:5) falling short. In dried blood spots CE(14:0), CE(16:1), LPC(20:4) and PC-O(40:5) which were not measured, however the remaining 7 measured lipids were all significantly associated with total concentration and passed multiple comparison correction based on bonferroni correct $p$-threshold $(p<0.0004)$ with

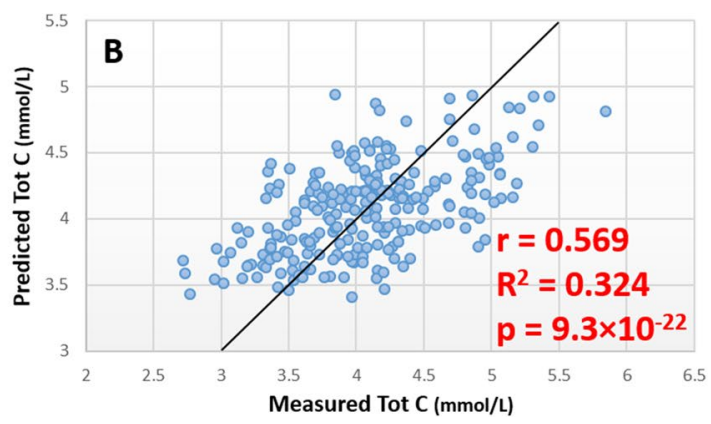

and tested in DBS 'validation' cohort using lipid markers identified in plasma

all of these lipids also exhibiting a stronger association than is observed between these lipids and total cholesterol concentration than is seen in the discovery cohort (Table S5).

\subsection{Stratification based on estimated lipoprotein concentration}

Plasma samples were stratified into the clinical risk categories based on their estimated lipoprotein concentration (Table 2). Total classification accuracies of $83.9 \%, 64.9 \%$, $82.2 \%$, and $64.9 \%$ were obtained for triglyceride, HDL, LDL and total cholesterol respectively (Table 2). Classification accuracy was not equal across the groups for any of the lipoproteins, with the risk category with the most samples in it having the highest accuracy with the exception of the 'low risk' total group which only contained 3 samples.

Stratification of DBS samples in the ABCD cohort was based on quartile rather than clinical cut-offs as the samples came from adolescents only $3 \%$ of the measurements fell outside of the healthy range. Classification accuracy was not equal across the quartiles with all of the 
lipoproteins showing better classification accuracy in the 1 st and 4th quartiles (Table 2).

\section{Discussion}

To our knowledge this is the first study to compare results obtained from DBSs and plasma samples in complex lipidomics studies and to determine that if we tried to answer the same biological question in both sample types would we achieve the same answer. Whilst we measured more lipids from the plasma samples (163 compared to 118) a high proportion $(71 \%)$ of the lipids measured in the DBS samples were also measured in plasma, with this lower number of lipids likely the result of less biological material being obtained from a single DBS punch than was extracted for the plasma samples.

Applying random forest machine learning to the lipid profile data generated from the plasma samples we were able to obtain good estimates $(r>0.7)$ of the concentration of triglyceride (12 lipid panel) and HDL (11 lipid panel) and modest estimates ( $r>0.4)$ of LDL (10 lipid panel) and total cholesterol (11 lipid panel) When these lipids were measured in an independent cohort of DBS samples 2 of the TG markers were not measured whilst the other 10 validated the association seen in the plasma samples. For HDL all 11 lipids validated, for LDL 2 were not measured and 8 validated and for total cholesterol 4 lipids were not measured in DBS while the remaining 7 lipids validated between cohorts. Using these panels of lipid biomarkers identified in plasma samples we were also able to use them to obtain a good estimate of triglyceride concentration and modest estimates of HDL, LDL and total cholesterol concentrations. This begins to suggest that dried blood spots can be used as an alternative to plasma samples and that the process of adding and removing the sample from the card is not altering the composition of the lipidome and its association with relationship with outcome measures, in this case the concentration of the four 'clinical lipids'. This finding is keeping with previous reports that have shown DBS as a viable alternative (Koulman et al. 2014; Mahajan et al. 2018) however this is the first study to directly this comparability.

We also wanted to see if the concentration estimates generated from both plasma and DBS samples were accurate enough to be used in clinical practice. Knowing a patients exact concentration of TriG, HDL, LDL or total cholesterol is less important than knowing their relative concentration (https://www.nhs.uk/conditions/high-chole sterol/cholesterol-levels/; Nantsupawat et al. 2019), i.e. if they fall outside of the healthy range, with a well-defined set of cut-offs described for each of the four "clinical lipids' (https://www.nhs.uk/conditions/high-cholesterol/ cholesterol-levels/; Nantsupawat et al. 2019). In plasma our estimated concentrations classified people into the correct clinical category with accuracies of $83.9 \%, 64.9 \%$, $82.2 \%$, and $64.9 \%$ for triglyceride, HDL, LDL and total cholesterol respectively. However, it is generally accepted that clinical biomarkers and test should have a sensitivity and specificity over 90\% ("Biomarkers: portents of malignancy"). So whilst these accuracies are all significantly higher than we would expect to see if we assigned individuals to groups randomly, but they are not good enough to be used in clinical practice. In the DBS cohort, looking at clinical cut-offs was inappropriate as the samples came from children with only $3 \%$ of the measurements falling outside of the healthy range, instead we looked at the relative distribution based on quartiles. As with plasma samples this is higher classification accuracy than we would expect if we assigned individuals to groups randomly but it still falls well short of the $90 \%$ sensitivity and specificity required for clinical practice.

Whilst the estimates that we have obtained in this study are not currently accurate enough for clinical use there does appear to be room for improvement. Looking at both plasma and DBS sample it can be seen that the risk category that most samples belong to being the most accurately classified as there was an overall bias with an significant over estimation of concentrations in this range (Table 2). This bias occurs because during the training phase the model identifies the optimum parameters to obtain the best prediction accuracy possible. So if the samples are clustered at the bottom of the concentration range, then the model will be biased towards predicting low concentrations as this will increase prediction accuracy for example in a situation where we have no information about a sample based on probability it is more likely to have a low concentration than a high one. Triglyceride has a more even distribution across the measure concentration range in both plasma and DBS (and as a result of this we for this lipoprotein we see a stronger correlation between estimated and measured concentration as well as a higher accuracy when assigning individuals to clinical groups relative to the other three lipoproteins in both plasma and DBS samples.

Whilst fewer lipids were measured in dried blood spots relative to plasma samples lipid markers used to predict the concentration of lipoproteins in plasma also predict their concentration when measured in DBS samples. This demonstrates that dried blood spots have the same association between the lipid profile of the sample and the clinical outcome.

Acknowledgements We would like to thank the JPI healthy diet for a healthy life and the BBSRC (BB/P028195/1) and the NIHR Cambridge Biomedical Research Centre (146281) for supporting this work. 
Author contributions Stuart G. Snowden was involved in the design of the study, performed all data analysis, and was primarily responsible for writing the manuscript, Aniko Korosi and Susanne de Rooji were involved in the design of the study and collection of the samples, Albert Koulman was involved in designing the study and writing the manuscript.

Funding This work was funded by JPI healthy diet for a healthy life and the BBSRC (BB/P028195/1) and the NIHR Cambridge Biomedical Research Centre (146281).

Availability of data and material Data is available by request from the cohort owner.

\section{Compliance with ethical standards}

Conflicts of interest The authors have no conflicts of interest.

Ethical approval Both studies described in this manuscript were approved by the local Medical Ethics committee and conducted in accordance with the Helsinki declaration.

Consent to participate All samples were collected in accordance with the guidelines of local Medical Ethics committee and conducted in accordance with the Helsinki declaration.

Consent for publication All authors have consented to the publication of this manuscript.

Open Access This article is licensed under a Creative Commons Attribution 4.0 International License, which permits use, sharing, adaptation, distribution and reproduction in any medium or format, as long as you give appropriate credit to the original author(s) and the source, provide a link to the Creative Commons licence, and indicate if changes were made. The images or other third party material in this article are included in the article's Creative Commons licence, unless indicated otherwise in a credit line to the material. If material is not included in the article's Creative Commons licence and your intended use is not permitted by statutory regulation or exceeds the permitted use, you will need to obtain permission directly from the copyright holder. To view a copy of this licence, visit http://creativecommons.org/licenses/by/4.0/.

\section{References}

Abhijit, K. R., Kusuma, K. S., Chokkanna, V., \& Kunnavil, R. (2016). Estimation of total cholesterol from a single dried blood spot new application for an age old technique! Indian Journal of Applied Research, 6(2), 54-59.

Abu-Rabie, P., \& Spooner, N. (2009). Direct quantitative bioanalysis of drugs in dried blood spot samples using a thin-layer chromatography mass spectrometer interface. Analytical Chemistry, 81(24), 10275-10284

Alfazil, A. A., \& Anderson, R. A. (2008). Stability of benzodiazepines and cocaine in blood spots stored on filter paper. Journal of Analytical Toxicology, 32, 512-515.

Chambers, M. C., Maclean, B., Burke, R., Amodei, D., Ruderman, D. L., Neumann, S., et al. (2012). A cross-platform toolkit for mass spectrometry and proteomics. Nature Biotechnology, 30(10), 918-920.
Chance, D. H., De Jesus, V. R., \& Spitzer, A. R. (2014). Clinical chemistry and dried blood spots: increasing laboratory utilization by improved understanding of quantitative challenges. Bioanalysis, 6(21), 2791-2794.

Corso, G., Papagni, F., Gelzo, M., Gallo, M., Barone, R., Graf, M., et al. (2016). Development and validation of an enzymatic method for total cholesterol analysis using whole blood spot. Journal of Clinical Laboratory Analysis, 30(5), 517-523.

Crimmins, E., Ki Kim, J., McCreath, H., Faul, J., Weir, D., \& Seeman, T. (2014). Validation of blood-based assays using dried blood spots for use in large population studies. Biodemography and Social Biology, 60(1), 38-48.

Harshfield, E. L., Koulman, A., ZiemekD, M. L., Fauman, E. B., Paul, D. S., Stacey, D., et al. (2019). An unbiased lipid phenotyping approach to study the genetic determinants of lipids and their association with coronary heart disease risk factors. Journal of Proteome Research, 18(6), 2397-2410.

Johannessen, A. (2010). Dried blood spots in HIV monitoring: applications in resource-limited settings. Bioanalysis, 2(11), 1893-1908.

Komada, K., Sugiyama, M., Vongphrachanh, P., Xeuatvongsa, A., Khamphaphongphane, B., Kitamura, T., et al. (2015). Seroprevalence of chronic hepatitis B, as determined from dried blood spots, among children and their mothers in central Lao People's Democratic Republic: A multistage, stratified cluster sampling survey. International Journal of Infectious Disease, 36, 21-26.

Koulman, A., Prentice, P., Wong, C. Y., Matthews, L., Bond, N. J., Eiden, M., Griffin, J. L., \& Dunger, D. B. (2014). The development and validation of a fast and robust dried blood spot based lipid profiling method to study infant metabolism. Metabolomics, 10, 1018-1025. An unbiased lipid phenotyping approach to study the genetic determinants of lipids and their association with coronary heart disease risk factors.

Kreuter, M. W., McQueen, A., Boyum, S., \& Fu, Q. (2016). Unmet basic needs and health intervention effectiveness in low-income populations. Preventive Medicine, 91, 70-75.

Kyle, J. E., Casey, C. P., Stratton, K. G., Zink, E. M., Kim, Y. M., Zheng, X., et al. (2017). Comparing identified and statistically significant lipids and polar metabolites in 15-year old serum and dried blood spot samples for longitudinal studies. Rapid Communications in Mass Spectrometry, 31, 447-456.

Lacher, D. A., Berman, L. E., Chen, T. C., \& Porter, K. S. (2013). Comparison of dried blood spot to venous methods for hemoglobin A1c, glucose, total cholesterol, high-density lipoprotein cholesterol, and C-reactive protein. Clinica Chimica Acta, 422, 54-58.

Lakshmy, R., Gupta, R., Prabhakaran, D., Snehi, U., \& Reddy, K. S. (2010). Utility of dried blood spots for measurement of cholesterol and triglyceride surveillance study. Journal of Diabetes and Science Technology, 4(2), 258-262.

Lakshmy, R., Mathur, P., \& Gupta, R. (2012). Measurement of cholesterol and triglycerides from a dried blood spot in an Indian Council of Medical Research-World Health Organisation multicentric study on risk factors for non-communicable diseases in India. Journal of Clinical Lipidology, 6, 33-41.

Lakshmy, R., Tarik, M., \& Abraham, R. A. (2014). Role of dried blood spots in health and disease diagnosis in older adults. Bioanalysis, 6(23), 3121-3131.

Li, W., \& Tse, F. L. (2010). Dried blood spot sampling in combination with LC-MS/MS for quantitative analysis of small molecules. Biomedical Chromatography, 24(1), 49-65.

Lumey, L. H., Ravelli, A. C., Wiessing, L. G., Koppe, J. G., Treffers, P. E., \& Stein, Z. A. (1993). The Dutch famine birth cohort study: Design, validation of exposure, and selected characteristics of subjects after 43 years follow-up. Paediatric and Perinatal Epidemiology, 7(4), 354-367. 
Mahajan, S., Choudhary, M. C., Kumar, G., \& Gupta, E. (2018). Evaluation of dried blood spot as an alternative sample collection method for hepatitis C virus RNA quantitation and genotyping using a commercial system. Virus Disease, 29(2), 141-146.

Manicke, N. E., Abu-Rabie, P., Spooner, N., Ouyang, Z., \& Cooks, R. G. (2011). Quantitative analysis of therapeutic drugs in dried blood spot samples by paper spray mass spectrometry: An avenue to therapeutic drug monitoring. Journal of American Society for Mass Spectrometry, 22, 1501-1507.

Miller, I. M., Lacher, D. A., Chen, T. C., Zipf, G. W., Gindi, R. M., Galinsky, A. M., et al. (2015). Collection and laboratory methods for dried blood spots for haemoglobin A1c and total abd highdensity lipoprotein cholesterol in population based survey. Clinical Chimica Acta, 44, 143-154.

Nantsupawat, N., Booncharoen, A., Wisetborisut, A., Jiraporncharoen, W., Pinyopornpanish, K., Chutarattanakul, L., et al. (2019). Appropriate Total cholesterol cut-offs for detection of abnormal LDL cholesterol and non-HDL cholesterol among low cardiovascular risk population. Lipids in Health and Disease, 18(1), 1-8.

Page-Sharp, M., Nunn, T., Salman, S., Moore, B. R., Batty, K. T., Davis, T. M. E., et al. (2016). Validation and application of a dried blood spot ceftriaxone assay. Antimicrobial Agents and Chemotherapy, 6(1), 14-23.

Prentice, P. M., Turner, C., Wong, M. C. Y., \& Dalton, R. N. (2013). Stability of metabolites in dried blood spots stored at different temperatures over a 2-year period. Bioanalysis, 5(12), 1507-1514.

Quraishi, R., Lakshmy, R., Prabhakaran, D., Mukhopadhyay, A. K., \& Jailkhani, B. (2006). Use of filter paper stored dried blood for measurement of triglycerides. Lipids Health Disease, 5, 20.

Samuelsson, L. B., Hall, M. H., McLean, S., Porter, J. H., Berkman, L., Marino, M., et al. (2015). Validation of biomarkers of CVD risk from dried blood spots in community-based research:
Methodologies and study-specific serum equivalencies. Biodemography and Social Biology, 61(3), 285-297.

Spooner, N., Anderson, K. D., Siple, J., Wickremsinhe, E. R., Xu, Y., \& Lee, M. (2019). Microsampling: Considerations for its use in pharmaceutical drug discovery and development. Bioanalysis, 11(10), 1015-1038.

Van der Elst, K. C. M., Span, L. F. R., van Hateren, K., Vermeulen, K. M., van der Werf, T. S., Greijdanus, B., et al. (2013). Dried blood spot analysis suitable for therapeutic drug monitoring of voriconazole, fluconazole, and posaconazole. Antimicrobial Agents and Chemotherapy, 57(10), 4999-5004.

van Eijsden, M., Vrijkotte, T. G. M., Gemke, R. J. B. J., \& van der Wal, M. F. (2011). Cohort profile: The amsterdam born children and their development (ABCD) study. International Journal of Epidemiology, 40(5), 1176-1183.

Wilhelm, A. J., den Burger, J. C. G., \& Swart, E. L. (2014). Therapeutic drug monitoring by dried blood spot: Progress to date and future directions. Clinical Pharmacokinetics, 53(11), 961-973.

Yassin, M. E. M., Eldaif, W. A., \& Elkhider, I. M. (2013). Evaluation of dried blood spots stored at different temperature for detection of HIV antibodies using different rapid tests. Surgery. https://doi. org/10.4172/2161-1076.S12-002.

Yin, P., Lehmann, R., \& Xu, G. (2015). Effects of pre-analytical processes on blood samples used in metabolomics studies. Analytical and Bioanalytical Chemistry, 407(17), 4879-4892.

Zakaria, R., Allen, K. J., Koplin, J. J., Roche, P., \& Greaves, R. F. (2016). Advantages and challenges of dried blood spot analysis by mass spectrometry across the total testing process. EJIFCC, 27(4), 288-317.

Publisher's Note Springer Nature remains neutral with regard to jurisdictional claims in published maps and institutional affiliations. 